\title{
Inaugural Editorial for Integrative Molecular Medicine
}

\author{
Masayoshi Yamaguchi* \\ Department of Hematology and Medical Oncology, Emory University School of Medicine, Atlanta, USA
}

I am pleased to announce the launch of Integrative Molecular Medicine, a peer-reviewed, international, fully open access journal that welcomes articles on all aspects of biomedicine and clinical practice, research, education and policy.

The journal aims to publish original research articles covering novel findings in molecular, biological, and biomedicine research by providing an insight on breakthrough discoveries in basic and clinical medicinal research, thereby lending a strong impetus to this important and rapidly developing field and helping to forge new links between clinicians and molecular biologists. The broad publishing spectrum of Integrative Molecular Medicine includes rare and common disorders from diagnosis to treatment. Examples of appropriate articles include reports of molecular diagnostic methods, medical bioinformatics, ethical, legal, and social implications, and novel approaches to clinical diagnosis.

Integrative Molecular Medicine will be a journal that demonstrates quality, integrity and timeliness. We will strive to ensure that Integrative Molecular Medicine the high standards and stands out among online open access journals. We aim, for example, to be indexed by Thomson Reuters and achieve an impact factor within short time as possible.

In terms of Quality-we are in the process of formation of an excellent team of associate editors and editorial board members. Each member of the team will be carefully selected. Integrative Molecular Medicine intends to publish highest quality articles which will be purely online or fully open access. The journal's mission is to publish articles that contribute to the art and science of biomedicine and which have a positive impact on health. As such, we will publish articles with scientific credibility and rigour, and coherence and clarity in the writing. Contributions do not need to be novel as confirmatory and replication studies will be considered, however the article must present new findings, which could include the reporting of negative findings.

In terms of Integrity-Integrative Molecular Medicine will operate according to the highest standards of authorship, peer review, editing and publishing.The process of publication and any problems or disputes arising throughout the process and after publication will be handled with fairness and equity according to the COPE guidelines.

In terms of Timeliness-we aim to provide a rapid route from submission to publication in Integrative Molecular Medicine: 4 weeks from submission to first decision and 3 weeks from acceptance to online publication. This will be achieved with the support of associate editors and intimate involvement of the editorial board in the peer reviewing process and final decision by the Editor-in-Chief. As the journal grows, the size of the reviewing panel will increase accordingly to ensure that submissions are processed as quickly as possible. As soon as articles are accepted and produced, they will appear online with a digital object identifier and be immediately available for sharing with peers and colleagues.

The benefits of the publication model that Integrative Molecular Medicine is adopting are also worth highlighting. The scientific community as a whole invests a great deal of time and energy in publication, and for this investment to achieve the maximum impact, articles need to be disseminated as widely as possible. We realize that publication quality and free access at the point of use come at a price. All articles published by Integrative Molecular Medicine are fully open access: freely available to read, download and share on publication. Articles are published under the terms of the Creative Commons Attribution License and copyright is retained by the author(s).

We are working diligently to automate the processing of manuscripts processing by using the editorial manager to ensure quick review process and make it transparent as possible, a goal that should be realizable within 3 months. In the meantime, we ask that all submissions to be sent to submissions@oatext.com. In addition, we will use e-mail preferentially in our communications with referees and authors and make sure to improve the efficiency of the editorial process.

We hope that you will find Integrative Molecular Medicine a useful outlet for your research and a useful source of information, evidence and debate. Our Aims and Scope and Guidelines for Authors are available to view. For further information visit the OA Text website.
Copyright: (C2014 Yamaguchi. This is an open-access article distributed under the terms of the Creative Commons Attribution License, which permits unrestricted use, distribution, and reproduction in any medium, provided the original author and source are credited.
Correspondence to: Dr. Masayoshi Yamaguchi, Department of Hematology and Medical Oncology, Emory University School of Medicine, 1365 C Clifton Road, NE, Atlanta, GA 30322, USA, E-mail: yamamasa1155@yahoo.co.jp

Received: July 12, 2014; Accepted: July 13, 2014; Published: July 17, 2014 